\title{
Another Proof of the Faithfulness of the Lawrence-Krammer Representation of the Braid Group $B_{3}$
}

\author{
Mohammad N. Abdulrahim and Mariam Hariri \\ Department of Mathematics, Beirut Arab University, P.O. Box 11-5020, Beirut 11072809, Lebanon \\ Correspondence should be addressed to Mohammad N. Abdulrahim,mna@bau.edu.lb
}

Received 16 March 2012; Accepted 6 May 2012

Academic Editors: P. Koshlukov, H. Li, S. Yang, and Y. Zhou

Copyright (c) 2012 M. N. Abdulrahim and M. Hariri. This is an open access article distributed under the Creative Commons Attribution License, which permits unrestricted use, distribution, and reproduction in any medium, provided the original work is properly cited.

The Lawrence-Krammer representation of the braid group $B_{n}$ was proved to be faithful for $n \geq 3$ by Bigelow and Krammer. In our paper, we give a new proof in the case $n=3$ by using matrix computations. First, we prove that the representation of the braid group $B_{3}$ is unitary relative to a positive definite Hermitian form. Then we show the faithfulness of the representation by specializing the indeterminates $q$ and $t$ to complex numbers on the unit circle rather than specializing them to real numbers as what was done by Krammer.

\section{Introduction}

A group is said to be linear if it admits a faithful representation into $G L_{n}(K)$ for some natural number $n$ and some field $K$. The question of faithfulness of the braid group has been the subject of research for a long time. It has been shown that the Burau representation is faithful for $n \leq 3$ and is not faithful for $n \geq 5$ [1]. However, the faithfulness of the Burau representation in the case $n=4$ is still unknown. In [2], Krammer proved that the Lawrence-Krammer representation is faithful in the case $n=4$, where he assumed that $t$ is a real number with $0<t<1$. After that, Bigelow [3] used topological methods that are very different from the algebraic methods used by Krammer to prove that the Lawrence-Krammer representation is faithful for all $n$. In his argument, he assumed that $q$ and $t$ are variables. Shortly after, Krammer [4] found a new proof that shows that this representation is faithful for all $n$ using algebraic methods, in which he interchanged the roles of $q$ and $t$ in [2] and assumed that $q$ is a real number with $0<q<1$.

Our work uses a different method that basically depends on matrix computations to show that the Lawrence-Krammer representation of $B_{3}$ is faithful. In our argument, we specialize the indeterminates $q$ and $t$ to nonzero complex numbers on the unit circle rather 
than specializing them to real numbers. For a long time, it is known that the braid group $B_{3}$ is linear (see [1]). What makes our approach different from the previous methods is the fact that our work is computational, which depends only on simple rules in linear algebra. Also, the difference between our work and the previous ones is in specializing $q$ and $t$ to nonzero complex numbers on the unit circle rather than to real numbers in the interval $[0,1]$ as in [2] or [4].

In Section 3, We show that if $q$ and $t$ are chosen to be appropriate algebraically independent complex numbers on the unit circle, then this representation is equivalent to a unitary representation. This will be a tool to find a necessary and sufficient condition for an element of $B_{3}$ possibly to belong to the kernel of the Lawrence-Krammer representation. method.

In Section 4, we prove the faithfulness of this representation by a purely computational

\section{Definitions}

Definition 2.1. The braid group on $n$ strands, $B_{n}$, is the abstract group with presentation $B_{n}=$ $\left\langle\sigma_{1}, \ldots, \sigma_{n-1}\right| \sigma_{i} \sigma_{i+1} \sigma_{i}=\sigma_{i+1} \sigma_{i} \sigma_{i+1}$ for $i=1,2, \ldots, n-2$, and $\sigma_{i} \sigma_{j}=\sigma_{j} \sigma_{i}$ if $\left.|i-j| \geq 2\right\rangle$. The generators $\sigma_{1}, \ldots, \sigma_{n-1}$ are called the standard generators of $B_{n}$.

The Lawrence-Krammer representation of braid groups is a representation of the braid group $B_{n}$ in $G L_{m}\left(\mathbb{Z}\left[q^{ \pm 1}, t^{ \pm 1}\right]\right)=\operatorname{Aut}\left(V_{0}\right)$, where $m=n(n-1) / 2$ and $V_{0}$ is the free module of rank $m$ over $\mathbb{Z}\left[q^{ \pm 1}, t^{ \pm 1}\right]$. The representation is denoted by $K(q, t)$. For simplicity, we write $K$ instead of $K(q, t)$.

Definition 2.2. With respect to $\left\{x_{i, j}\right\}_{1 \leq i<j \leq n}$, the free basis of $V_{0}$, the image of each Artin generator under Krammer's representation is written as

$$
K\left(\sigma_{k}\right)\left(x_{i, j}\right)= \begin{cases}t q^{2} x_{k, k+1}, & i=k, j=k+1, \\ (1-q) x_{i, k}+q x_{i, k+1}, & j=k, i<k, \\ x_{i, k}+t q^{k-i+1}(q-1) x_{k, k+1}, & j=k+1, i<k, \\ t q(q-1) x_{k, k+1}+q x_{k+1, j}, & i=k, k+1<j, \\ x_{k, j}+(1-q) x_{k+1, j}, & i=k+1, k+1<j, \\ x_{i, j}, & i<j<k \text { or } k+1<i<j, \\ x_{i, j}+t q^{k-i}(q-1)^{2} x_{k, k+1}, & i<k<k+1<j .\end{cases}
$$

Using the Magnus representation of subgroups of the automorphism group of a free group with three generators, we determine Krammer's representation $K(q, t): B_{3} \rightarrow$ $G L_{3}\left(\mathbb{Z}\left[q^{ \pm 1}, t^{ \pm 1}\right]\right)$, where

$$
K\left(\sigma_{1}\right)=\left(\begin{array}{ccc}
t q^{2} & 0 & 0 \\
t q(q-1) & 0 & q \\
0 & 1 & 1-q
\end{array}\right), \quad K\left(\sigma_{2}\right)=\left(\begin{array}{ccc}
1-q & q & 0 \\
1 & 0 & t q^{2}(q-1) \\
0 & 0 & t q^{2}
\end{array}\right)
$$

Here, $\mathbb{Z}\left[q^{ \pm 1}, t^{ \pm 1}\right]$ is the ring of Laurent polynomials on two variables. For simplicity, we denote $K\left(\sigma_{i}\right)$ by $X_{i}$, where $i=1,2$. Here, $X_{i}^{*}$ is the conjugate transpose of $X$, where $\bar{t}=t^{-1}$ and $\bar{q}=q^{-1}$. 


\section{The Lawrence-Krammer Representation of $B_{3}$ Is Equivalent to a Unitary Representation}

Budney has proved that the L-K representation of the braid group $B_{n}$ is unitary relative to a hermitian form. His hermitian form turns out to be negative-definite (see [5]). In our work, we specialize the indeterminates $q$ and $t$ to nonzero complex numbers on the unit circle. We consider the complex representation $K: B_{3} \rightarrow G L_{3}(\mathbb{C})$ and prove that it is unitary relative to a positive definite Hermitian form.

Theorem 3.1. The images of the generators of $B_{3}$ under the Lawrence-Krammer representation are unitary relative to a Hermitian matrix $M$ given by

$$
M=\left(\begin{array}{ccc}
\frac{\left(1+q^{2}\right)(1+t q)\left(t q^{2}-1\right)}{t q^{2}(q-1)} & \frac{\left(1+q^{2}\right)(1-t q(q-1))}{t q^{2}} & 1+q^{2} \\
\frac{\left(1+q^{2}\right)\left(t q^{2}+q-1\right)}{q^{2}} & \frac{\left(1+q^{2}\right)\left(-1+t\left(1-(q-2)^{2} q+t q^{3}\right)\right)}{t q^{2}(q-1)} & \frac{\left(1+q^{2}\right)\left(t q^{2}+q-1\right)}{q} \\
\frac{q^{2}+1}{q^{2}} & \frac{\left(1+q^{2}\right)(1-t q(q-1))}{t q^{3}} & \frac{\left(1+q^{2}\right)(1+t q)\left(t q^{2}-1\right)}{t q^{2}(q-1)}
\end{array}\right) .
$$

That is, $X_{i} M X_{i}^{*}=M$ for $i=1,2$, where $M^{*}=M$.

The choice of $M$ is unique only if we specialize the indeterminates $q$ and $t$ in a way that Krammer's representation becomes irreducible. A necessary and sufficient condition for irreducibility is given in [6].

Our objective is to show that a certain specialization $M^{\prime}$ of $M$ is equivalent to the identity matrix, that is, $U M^{\prime} U^{*}=I$ for some matrix $U$. In other words, we need to show that $M^{\prime}=V V^{*}$ for some matrix $V$; or equivalently, $M^{\prime}$ is hermitian.

Theorem 3.2. Let $q$ and $t$ be nonzero complex numbers on the unit circle such that $q=e^{i \theta}$ and $t=e^{i \alpha}$. The matrix $M$ is positive definite when $\theta$ and $\alpha$ are chosen in either one of the following intervals:

$$
\begin{aligned}
& \text { (i) }-\pi / 2<\theta<0 \text { and }-\pi-(3 \theta / 2)<\alpha<-(3 \theta / 2) \text {, } \\
& \text { (ii) } 0<\theta<\pi / 2 \text { and }-(3 \theta / 2)<\alpha<\pi-(3 \theta / 2) \text {. }
\end{aligned}
$$

Proof. We denote the principal minors of $M$ by $d_{i}$, where $i=1,2,3$. We have

$$
\begin{aligned}
& d_{1}=\frac{\left(1+q^{2}\right)(1+t q)\left(t q^{2}-1\right)}{t q^{2}(q-1)}, \\
& d_{2}=\frac{\left(1+q^{2}\right)^{2}\left(t^{2} q^{3}-1\right)(-1+t q(-2+q(2+t q)))}{t^{2} q^{4}(q-1)^{2}}, \\
& d_{3}=\frac{\left(1+q^{2}\right)^{3}(1+t)\left(t q^{3}-1\right)\left(t^{2} q^{3}-1\right)^{2}}{t^{3} q^{6}(q-1)^{3}} .
\end{aligned}
$$


We find the intervals of $q$ and $t$ for which $d_{1}, d_{2}$, and $d_{3}$ are strictly positive. Let $q=e^{i \theta}$ and $t=e^{i \alpha}$, where $-\pi \leq \theta \leq \pi$ and $-\pi \leq \alpha \leq \pi$. Then we have

$$
\begin{aligned}
& d_{1}=2 \cos \theta\left[1+\csc \frac{\theta}{2} \sin \left(\frac{3 \theta}{2}+\alpha\right)\right], \\
& d_{2}=4 \cos ^{2} \theta \csc \frac{\theta}{2} \sin \left(\frac{3 \theta}{2}+\alpha\right)\left[2+\csc \frac{\theta}{2} \sin \left(\frac{3 \theta}{2}+\alpha\right)\right], \\
& d_{3}=8 \cos ^{3} \theta \csc ^{3} \frac{\theta}{2} \sin ^{2}\left(\frac{3 \theta}{2}+\alpha\right)\left[\sin \frac{3 \theta}{2}+\sin \left(\frac{3 \theta}{2}+\alpha\right)\right] .
\end{aligned}
$$

In order to have $d_{1}, d_{2}$, and $d_{3}$ strictly positive real numbers, we consider the intervals given by the hypothesis and make the following observations.

In the interval (i), it is easy to see that $\csc (\theta / 2)<0, \sin (3 \theta / 2+\alpha)<0, \cos \theta>0$, and $\sin (3 \theta / 2)+\sin (3 \theta / 2+\alpha)<0$.

In the interval (ii), we see that $\csc (\theta / 2)>0, \sin (3 \theta / 2+\alpha)>0, \cos \theta>0$, and $\sin (3 \theta / 2)+$ $\sin (3 \theta / 2+\alpha)>0$.

We construct a homomorphism that specializes the indeterminates $q$ and $t$ to nonzero complex numbers, on the unit circle, which are transcendentally independent over $\mathbb{Z}$.

Let $f_{w}$ be a homomorphism $f_{w}: \mathbb{Z}\left[q^{ \pm 1}, t^{ \pm 1}\right] \rightarrow \mathbb{C}$ defined as follows: $f_{w}(q)=w_{1}$, $f_{w}(t)=w_{2}, f_{w}(z)=z$ for $z \in \mathbb{Z}$, where $w=\left(w_{1}, w_{2}\right)$ and $w_{1}, w_{2}$ are complex numbers on the unit circle. Let $f_{w}$ also denote the group homomorphism $G L_{3}\left(\mathbb{Z}\left[q^{ \pm 1}, t^{ \pm 1}\right]\right) \rightarrow G L_{3}(\mathbb{C})$. We choose $w$ such that $f_{w}(M)$ is positive definite; that is, $f_{w}(M)=V V^{*}$, for some $V \in G L_{3}(\mathbb{C})$.

Consider now the composition map $f_{w} \circ K: B_{3} \rightarrow G L_{3}(\mathbb{C})$. It was proved in [6] that the specialization of Krammer's representation $B_{3} \rightarrow G L_{3}(\mathbb{C})$ is irreducible if and only if $t \neq-1$, $t q^{3} \neq 1$, and $t^{2} q^{3} \neq 1$. It is easy to see that the conditions for irreducibility are achieved once we choose $q$ and $t$ in either one of the intervals (i) or (ii) in the hypothesis of Theorem 3.2. The uniqueness of $f_{w}(M)$ up to scalar multiplication follows from Shur's Lemma and the fact that the specialization of Krammer's representation of $B_{3}$ is irreducible.

Theorem 3.3. The complex representation of $B_{3}, f_{w} \circ K$, is conjugate to a unitary representation.

Proof. Since $X_{i} M X_{i}^{*}=M$ for $i=1,2$, it follows that

$$
f_{w}\left(X_{i}\right) f_{w}(M) f_{w}\left(X_{i}^{*}\right)=f_{w}(M) .
$$

But $f_{w}(M)=V V^{*}$, then

$$
\left(V^{-1} f_{w}\left(X_{i}\right) V\right)\left(V^{-1} f_{w}\left(X_{i}\right) V\right)^{*}=I .
$$

Now, let

$$
U=V^{-1} f_{w}\left(X_{i}\right) V,
$$

then

$$
U U^{*}=I .
$$

Hence $U^{*} U=I$ and so $U$ is unitary. 
We now find a necessary and sufficient condition for an element of $B_{3}$ possibly to belong to the kernel of the Lawrence-Krammer representation.

Theorem 3.4. An element of $B_{3}$ lies in the kernel of the Lawrence-Krammer representation of $B_{3}$ if and only if the trace of its image is equal to three.

Proof. If $\operatorname{tr}\left(f_{w}\left(X_{i}\right)\right)=3$, then $\operatorname{tr}(U)=3$, where $U=V^{-1} f_{w}\left(X_{i}\right) V$. Since $U$ is unitary, it follows that $U$ is diagonalizable, that is, there exists a matrix $P$ such that $P^{-1} U P=D$, where $D$ is a diagonal matrix with eigenvalues of $U$ as the diagonal entries. Hence $\lambda_{1}+\lambda_{2}+\lambda_{3}=3$, where the $\lambda_{i}^{\prime} s$ are the eigenvalues of $U$. Being unitary, it has its eigenvalues on the unit circle. Therefore, we get $\lambda_{1}=\lambda_{2}=\lambda_{3}=1$. Thus, $D$ is the identity matrix and so is $U$. This implies that $f_{w}\left(X_{i}\right)=I_{3}$.

Therefore, we conclude that if there exists a nontrivial element in $B_{3}$ such that the trace of its image under $f_{w} \circ K: B_{3} \rightarrow G L_{3}(\mathbb{C})$ is three then, the element lies in the kernel of this representation.

\section{The Faithfulness of the L-K Representation for $n=3$}

In this section, we prove our main theorem, which shows the faithfulness of our representation for certain values of $q$ and $t$. Let $q$ and $t$ be nonzero complex numbers on the unit circle such that $q=e^{i \theta}$ and $t=e^{i \alpha}$.

Let

$\beta=\arg \left(\frac{1+t q^{2}+t^{2} q^{4}-q(1+t)-q^{3} t(1+t)+\sqrt{-4 q^{4} t^{2}+\left(1+(-1+q(q-1) t)\left(1+t q^{2}\right)\right)^{2}}}{2 t q^{2}}\right)$.

Consider the values of $q$ and $t$ whose arguments belong to the set $G$ defined as follows:

$$
G=\left\{(\theta, \alpha) \in A \cup B \mid \theta \neq \pm \frac{\pi}{3}, \frac{\alpha+3 \theta}{\pi} \notin \mathbb{Q}, \frac{\beta}{\pi} \notin \mathbb{Q}\right\}
$$

where

$$
\begin{gathered}
A=\left\{(\theta, \alpha) \mid-\frac{\pi}{2}<\theta<0,-\frac{\pi}{3}-2 \theta<\alpha<-\frac{3 \theta}{2}\right\}, \\
B=\left\{(\theta, \alpha) \mid 0<\theta<\frac{\pi}{2},-\frac{3 \theta}{2}<\alpha<\frac{\pi}{3}-2 \theta\right\} .
\end{gathered}
$$

The set $G$ is a subset of the union of the intervals given in Theorem 3.2. We choose $w$ such that $(\theta, \alpha) \in G$. Therefore, for values of $q$ and $t$ such that $(\theta, \alpha) \in G$, we have $f_{w}(M)$ being positive definite and $f_{w} \circ K: B_{3} \rightarrow G L_{3}(\mathbb{C})$ irreducible. For simplicity, we still write $K$ instead of $f_{w} \circ K$.

We now present our second main theorem. 
Theorem 4.1. The complex specialization of the Lawrence-Krammer representation $\mathrm{K}: \mathrm{B}_{3} \rightarrow$ $G L_{3}(\mathbb{C})$ is faithful for a dense subset of the set $G$ defined above.

We need to state some lemmas that help us prove Theorem 4.1. We already know that $B_{3}=\left\langle\sigma_{1}, \sigma_{2} \mid \sigma_{1} \sigma_{2} \sigma_{1}=\sigma_{2} \sigma_{1} \sigma_{2}\right\rangle$, where $\sigma_{1}$ and $\sigma_{2}$ are called the standard generators of $B_{3}$. Consider the product of generators of $B_{3}$, namely, $J=\sigma_{1} \sigma_{2}$. We also let $S=\sigma_{1} J$. It was proved in [7] that $B_{3}$ is generated by the two elements $S$ and $J$ and the relation $S^{2}=J^{3}$. That is, we have that $B_{3}=\left\langle S, J \mid S^{2}=J^{3}\right\rangle$. Also, it was proved in [8] that the center $Z\left(B_{3}\right)$ of $B_{3}$ is generated by the one generator $J^{3}$, that is, $Z\left(B_{3}\right)=\left\langle J^{3}\right\rangle$. Thus, the elements of $B_{3}$ are of the form: $S^{l}, J^{m}, J^{m_{1}} S^{l_{1}} J^{m_{2}} S^{l_{2}}, \ldots$, and so forth, where all the exponents are integers.

Consider the element $S^{l}$. If $l$ is odd, then $S^{l}=S^{2 k+1}=S \cdot S^{2 k}=S J^{3 k}$, where $k \in \mathbb{Z}$. If $l$ is even then $S^{l}=S^{2 k}=J^{3 k}$, where $k \in \mathbb{Z}$. Therefore, any element of $B_{3}$ is of one of the following forms: $S, J^{m}, S J^{m_{1}} S J^{m_{2}} \ldots$, or $J^{m_{1}} S J^{m_{2}} S \ldots$, where $m_{i}$ 's $\in \mathbb{Z}$.

Lemma 4.2. The possible nontrivial elements in the kernel of the representation $K: B_{3} \rightarrow G L_{3}(\mathbb{C})$ are

(i) $S J^{r_{1}} S J^{r_{2}} \cdots S J^{r_{2 n-1}} S J^{-3 n-\sum_{i=1}^{2 n-1} r_{i}}$, where the number of $S^{\prime} s$ is $2 n$ and $r_{i}=1$ or 2 for $1 \leq$ $i<2 n$,

(ii) elements obtained from (i) by permuting $S$ and $J$.

Proof. Recall that $q=e^{i \theta}, t=e^{i \alpha}$, and $K\left(\sigma_{i}\right)=X_{i}$, where $i=1,2$. For simplicity, we use the symbol $|X|$ instead of $\operatorname{det}(X)$. We have that $J=\sigma_{1} \sigma_{2}, S=\sigma_{1} J$, and $\left|X_{1}\right|=\left|X_{2}\right|=-t q^{3}$. In general, elements of $B_{3}$ are of the form: $S, J^{m}, S J^{m_{1}} S J^{m_{2}} \cdots S J^{m_{k}}$, or elements obtained by permuting $S$ and $J$ in the element $S J^{m_{1}} S J^{m_{2}} \ldots S J^{m_{k}}$. We deal with each of these forms separately.

Consider the element $S$. We have $|K(S)|=\left|X_{1}\right|^{3}=\left(-t q^{3}\right)^{3}$. Assume that $|K(S)|=1$. Then $\left(t q^{3}\right)^{6}=1$ and so $6(\alpha+3 \theta)=2 c \pi, c \in \mathbb{Z}$. This implies that $(\alpha+3 \theta) / \pi=(c / 3) \in \mathbb{Q}$, which is a contradiction when $(\theta, \alpha) \in G$. Therefore, $|K(S)| \neq 1$ and so $S \notin \operatorname{Ker} K$.

Consider the element $J^{m}$, where $m \neq 0$. We have $\left|K\left(J^{m}\right)\right|=\left|X_{1}\right|^{2 m}=\left(t q^{3}\right)^{2 m}$. Assume that $\left|K\left(J^{m}\right)\right|=1$. Then $\left(t q^{3}\right)^{2 m}=1$ and so $2 m(\alpha+3 \theta)=2 c \pi, c \in \mathbb{Z}$. This implies that $(\alpha+$ $3 \theta) / \pi=(c / m) \in \mathbb{Q}$, which is a contradiction when $(\theta, \alpha) \in G$. Therefore, $\left|K\left(J^{m}\right)\right| \neq 1$ and so $J^{m} \notin \operatorname{Ker} K$.

Consider the element $u=S J^{m_{1}} S J^{m_{2}} \ldots S J^{m_{2 n+1}}$, where the number of $S^{\prime} \mathrm{s}$ is $2 n+1$. We have $|K(u)|=\left|X_{1}\right|^{h}$, where $h=3(2 n+1)+2 \sum_{i=1}^{2 n+1} m_{i} \in \mathbb{Z}$. It is clear that we have $h \neq 0$. Assume that $|K(u)|=1$. Then $\left|X_{1}\right|^{h}=\left(-t q^{3}\right)^{h}=1$ and so $\left(t q^{3}\right)^{2 h}=1$. Then $2 h(\alpha+3 \theta)=2 c \pi, c \in \mathbb{Z}$. This implies that $(\alpha+3 \theta) / \pi=(c / h) \in \mathbb{Q}$, which is a contradiction when $(\theta, \alpha) \in G$. Therefore, $|K(u)| \neq 1$, that is, $u \notin$ Ker $K$. It follows, by Theorem 3.4, that $\operatorname{tr}(K(u)) \neq 3$. Now, consider an element $v$ obtained by permuting $S$ and $J$ in $u$. Then $v$ is an element with odd number of $S^{\prime}$ s. The trace of the image of $V$ is equal to that of the image of an element having the same form as $u$. This implies that $\operatorname{tr}(K(v)) \neq 3$ and thus $v \notin$ Ker $K$.

Consider the element $u=S J^{m_{1}} S J^{m_{2}} \cdots S J^{m_{2 n}}$, where the number of $S^{\prime}$ s is $2 n$. Then $|K(u)|=\left|X_{1}\right|^{h}$, where $h=2\left(3 n+\sum_{i=1}^{2 n} m_{i}\right) \in \mathbb{Z}$. For $u \in \operatorname{Ker} K$, we have $|K(u)|=1$, that is, $\left(-t q^{3}\right)^{h}=1$. If $h \neq 0$, then $\left(t q^{3}\right)^{2 h}=1$ and so $2 h(\alpha+3 \theta)=2 c \pi, c \in \mathbb{Z}$. This implies that $(\alpha+3 \theta) / \pi=(c / h) \in \mathbb{Q}$, which contradicts the fact that $(\theta, \alpha) \in G$. Thus $h=0$, that is, $\sum_{i=1}^{2 n} m_{i}=-3 n$. We write $m_{2 n}=-3 n-\sum_{i=1}^{2 n-1} m_{i}$. Then $u=S J^{m_{1}} S J^{m_{2}} \cdots S J^{m_{2 n-1}} S J^{-3 n-\sum_{i=1}^{2 n-1} m_{i}}$.

If $m_{i}=3 k_{i}$ for some $1 \leq i<2 n$, then $J^{m_{i}} \in Z\left(B_{3}\right)$. It follows that $S J^{m_{i}}=J^{m_{i}} S$. Using the relation $S^{2}=J^{3}, u$ will be reduced to an element with less number of $S^{\prime} s$ and $m_{i} \neq 3 k_{i}$ for all $i$. 
In the case $m_{i} \neq 3 k_{i}$ for every $1 \leq i<2 n$, we have $m_{i}=3 k_{i}+r_{i}$, where $r_{i}=1$ or 2 . Then $u=S J^{3 k_{1}+r_{1}} \cdots S J^{3 k_{2 n-1}+r_{2 n-1}} S J^{-3 n-\left(3 k_{1}+r_{1}+3 k_{2}+r_{2}+\cdots+3 k_{2 n-1}+r_{2 n-1}\right)}$. Since $J^{3 k_{i}} \in Z\left(B_{3}\right)$, it follows that $u=S J^{r_{1}} S J^{r_{2}} \cdots S J^{r_{2 n-1}} S J^{-3 n-\sum_{i=1}^{2 n-1} r_{i}}$, where $r_{i}=1$ or 2 . Since $u \in \operatorname{Ker} K$, it follows that the trace of the image of $u$ is equal to 3. Consider an element $v$ obtained by permuting $S$ and $J$ in the element $u$. Then $v$ is an element that has an even number of $S^{\prime}$ s. The trace of the image of $V$ is equal to that of an element having the same form as $u$. This implies that $\operatorname{tr}(K(v))=3$. It follows, by Theorem 3.4, that $v \in \operatorname{Ker} K$.

Therefore, the possible nontrivial elements in the kernel of $K: B_{3} \rightarrow G L_{3}(\mathbb{C})$ are $S J^{r_{1}} S J^{r_{2}} \ldots S J^{r_{2 n-1}} S J^{-3 n-\sum_{i=1}^{2 n-1} r_{i}}$, and other elements that are obtained by permuting $S$ and $J$. Here, the number of $S^{\prime} \mathrm{s}$ is $2 n$ and $r_{i}=1$ or 2 for $1 \leq i<2 n$.

Now, we present a lemma that will be used in the proof of Theorem 4.1.

\section{Lemma 4.3.}

$$
K\left(J^{n}\right)= \begin{cases}\left(t^{2} q^{6}\right)^{k} I, & n=3 k \\ \left(t^{2} q^{6}\right)^{k} K(J), & n=3 k+1, \\ \left(t^{2} q^{6}\right)^{k} K\left(J^{2}\right), & n=3 k+2 .\end{cases}
$$

Here $k \in \mathbb{N}$ and I is the identity matrix.

Proof. Direct computations show that

$$
\begin{gathered}
K(J)=\left(\begin{array}{ccc}
-t q^{2}(q-1) & t q^{3} & 0 \\
-t q(q-1)^{2} & t q^{2}(q-1) & t q^{3} \\
1 & 0 & 0
\end{array}\right), \\
K\left(J^{2}\right)=\left(\begin{array}{ccc}
0 & 0 & t^{2} q^{6} \\
t q^{3} & 0 & t^{2} q^{5}(q-1) \\
-t q^{2}(q-1) & t q^{3} & 0
\end{array}\right), \\
K\left(J^{3}\right)=\left(t^{2} q^{6}\right) I, \quad K\left(J^{4}\right)=\left(t^{2} q^{6}\right) K(J), \quad K\left(J^{5}\right)=\left(t^{2} q^{6}\right) K\left(J^{2}\right) .
\end{gathered}
$$

Using mathematical induction on the integer $k$, we prove the lemma.

\section{Proof of Theorem 4.1}

In order to prove that the representation $K: B_{3} \rightarrow G L_{3}(\mathbb{C})$ is faithful, we have to show that the elements in Lemma 4.2 do not belong to $\operatorname{Ker} K$ if we choose $q$ and $t$ in a way that their arguments $\theta$ and $\alpha$ belong to the previously defined set $G$.

That is, we need to show that the element $u=S J^{r_{1}} S J^{r_{2}} \ldots S J^{r_{2 n-1}} S J^{-3 n-\sum_{i=1}^{2 n-1} r_{i}}$ and others obtained from $u$ by permuting $S$ and $J$ do not belong to the kernel of this representation if $(\theta, \alpha) \in G$.

Consider the element $u=S J^{r_{1}} S J^{r_{2}} \ldots S J^{r_{2 n-1}} S J^{-3 n-\sum_{i=1}^{2 n-1} r_{i}}$, where the number of $S^{\prime} \mathrm{s}$ is $2 n$ and $r_{i}=1$ or 2 for $1 \leq i<2 n$. We consider five cases and we prove that the element $u$, in each of the cases, does not belong to the kernel of the complex specialization $K: B_{3} \rightarrow G L_{3}(\mathbb{C})$. 
(1) If $r_{i}=1$ for all $1 \leq i<2 n$, then

$$
u=\left(S J^{1}\right)^{2 n-1} S J^{-3 n-(2 n-1)}=\left(S J^{1}\right)^{2 n-1} S J^{-3(2 n-1)+n-2} .
$$

Since $J^{-3} \in Z\left(B_{3}\right)$, it follows that $u=\left(S J^{-2}\right)^{2 n-1} S J^{n-2}$ and so $u=\left(S J^{-2}\right)^{2 n} J^{n}$. The eigenvalues of $K\left(S J^{-2}\right)$ are $1,-1 / q$ and $1 / t q^{2}$, which are easily shown to be distinct for $(\theta, \alpha) \in G$. We diagonalize $K\left(S J^{-2}\right)$ by a matrix $T$ given by

$$
T=\left(\begin{array}{ccc}
t q^{2} & -t q^{3} & t^{2} q^{4} \\
t q(q-1) & -t q^{2}(q-1) & \frac{q(-1+t q(-1+q+q(1+(q-1) q) t))}{q-1} \\
1 & 1 & 1
\end{array}\right)
$$

It is easy to see that $\operatorname{det}(T)=-\left(q^{3}(1+q) t(1+t q)\left(t q^{2}-1\right)\right) /(q-1) \neq 0$ for $(\theta, \alpha) \in G$.

Then

$$
\begin{aligned}
T^{-1} K\left(S J^{-2}\right) T & =\left(\begin{array}{ccc}
1 & 0 & 0 \\
0 & -\frac{1}{q} & 0 \\
0 & 0 & \frac{1}{t q^{2}}
\end{array}\right) \\
T^{-1} K(u) T & =\left(\begin{array}{ccc}
1 & 0 & 0 \\
0 & \frac{1}{q^{2 n}} & 0 \\
0 & 0 & \frac{1}{\left(t q^{2}\right)^{2 n}}
\end{array}\right) T^{-1} K\left(J^{n}\right) T .
\end{aligned}
$$

We take the following possibilities regarding the value of $n$.

(a) For $n=3 k$, we have, from Lemma 4.3, that $T^{-1} K\left(J^{n}\right) T=\left(t^{2} q^{6}\right)^{k} I$. Then we get

$$
T^{-1} K(u) T=\left(\begin{array}{ccc}
\left(t^{2} q^{6}\right)^{k} & 0 & 0 \\
0 & t^{2 k} & 0 \\
0 & 0 & \frac{1}{\left(t^{4} q^{6}\right)^{k}}
\end{array}\right)
$$

The entry $\left(t^{2} q^{6}\right)^{k} \neq 1$; otherwise, $e^{i k(2 \alpha+6 \theta)}=1$. That is, $(\alpha+3 \theta) / \pi \in \mathbb{Q}$, which contradicts the fact that $(\theta, \alpha) \in G$.

(b) For $n=3 k+1$, we have, from Lemma 4.3, that $T^{-1} K\left(J^{n}\right) T=\left(t^{2} q^{6}\right)^{k} T^{-1} K(J) T$. Direct computations give

$$
T^{-1} K(u) T=\left(\begin{array}{ccc}
* & \frac{t q^{3}\left(t^{2} q^{6}\right)^{k}\left(1-t q^{3}\right)}{(1+q)\left(t q^{2}-1\right)} & * \\
* & * & * \\
* & * & *
\end{array}\right)
$$


It is easy to show that $\left(t q^{3}\left(t^{2} q^{6}\right)^{k}\left(1-t q^{3}\right)\right) /\left((1+q)\left(t q^{2}-1\right)\right) \neq 0$ for $(\theta, \alpha) \in G$.

(c) For $n=3 k+2$, we have, from Lemma 4.3, that $T^{-1} K\left(J^{n}\right) T=\left(t^{2} q^{6}\right)^{k} T^{-1} K\left(J^{2}\right) T$. We get, by direct computations,

$$
T^{-1} K(u) T=\left(\begin{array}{ccc}
* & \frac{t q^{4}\left(t^{2} q^{6}\right)^{k}\left(t q^{3}-1\right)}{(1+q)\left(t q^{2}-1\right)} & * \\
* & * & * \\
* & * & *
\end{array}\right) .
$$

It is easy to show that $\left(t q^{4}\left(t^{2} q^{6}\right)^{k}\left(t q^{3}-1\right)\right) /\left((1+q)\left(t q^{2}-1\right)\right) \neq 0$ for $(\theta, \alpha) \in G$.

(2) If $r_{i}=2$ for all $1 \leq i<2 n$, then

$$
u=\left(S J^{2}\right)^{2 n-1} S J^{-3 n-2(2 n-1)}=\left(S J^{2}\right)^{2 n-1} S J^{-3(2 n-1)-n-1} .
$$

Since $J^{-3} \in Z\left(B_{3}\right)$, it follows that $u=\left(S J^{-1}\right)^{2 n-1} S J^{-n-1}$ and so $u=\left(S J^{-1}\right)^{2 n} J^{-n}$. The eigenvalues of $K\left(S J^{-1}\right)$ are $1,-q$, and $t q^{2}$. It is easy to show that these eigenvalues are distinct when $(\theta, \alpha) \in G$. We diagonalize $K\left(S J^{-1}\right)$ by a matrix $H$ given by

$$
H=\left(\begin{array}{ccc}
0 & 0 & \frac{(1+t q)\left(t q^{2}-1\right)}{t(q-1)} \\
q & -1 & -1+q+t q^{2} \\
1 & 1 & 1
\end{array}\right)
$$

It is clear that $\operatorname{det}(H)=\left((1+q)(1+t q)\left(t q^{2}-1\right)\right) /(t(q-1)) \neq 0$ for $(\theta, \alpha) \in G$.

Then

$$
\begin{gathered}
H^{-1} K\left(S J^{-1}\right) H=\left(\begin{array}{ccc}
1 & 0 & 0 \\
0 & -q & 0 \\
0 & 0 & t q^{2}
\end{array}\right), \\
H^{-1} K(u) H=\left(\begin{array}{ccc}
1 & 0 & 0 \\
0 & q^{2 n} & 0 \\
0 & 0 & \left(t q^{2}\right)^{2 n}
\end{array}\right) H^{-1} K\left(J^{-n}\right) H .
\end{gathered}
$$


Using Lemma 4.3, we can easily see that

$$
K\left(J^{-n}\right)= \begin{cases}\frac{1}{\left(t^{2} q^{6}\right)^{k}} I, & n=3 k, \\ \frac{1}{\left(t^{2} q^{6}\right)^{k}} K\left(J^{-1}\right), & n=3 k+1, \\ \frac{1}{\left(t^{2} q^{6}\right)^{k}} K\left(J^{-2}\right), & n=3 k+2 .\end{cases}
$$

Here $k$ is a positive integer. We deal with the following possibilities regarding the value of $n$.

(a) For $n=3 k$, a comuptation shows that

$$
H^{-1} K(u) H=\left(\begin{array}{ccc}
\frac{1}{\left(t^{2} q^{6}\right)^{k}} & 0 & 0 \\
0 & \frac{1}{t^{2 k}} & 0 \\
0 & 0 & \left(t^{4} q^{6}\right)^{k}
\end{array}\right)
$$

The entry $1 /\left(t^{2} q^{6}\right)^{k} \neq 1$; otherwise, $e^{i k(2 \alpha+6 \theta)}=1$, which implies that $(\alpha+3 \theta) / \pi \in \mathbb{Q}$. This contradicts the fact that $(\theta, \alpha) \in G$.

(b) For $n=3 k+1$, a computation shows that

$$
H^{-1} K(u) H=\left(\begin{array}{ccc}
* & 1-t q^{3} & * \\
& t q^{3}\left(t^{2} q^{6}\right)^{k}(1+q)\left(t q^{2}-1\right) & \\
* & * & * \\
* & * & *
\end{array}\right) .
$$

It is easy to show that $\left(1-t q^{3}\right) /\left(t q^{3}\left(t^{2} q^{6}\right)^{k}(1+q)\left(t q^{2}-1\right)\right) \neq 0$ for $(\theta, \alpha) \in G$.

(c) For $n=3 k+2$, a computation shows that

$$
H^{-1} K(u) H=\left(\begin{array}{ccc}
* & t q^{3}-1 & * \\
& t q^{4}\left(t^{2} q^{6}\right)^{k}(1+q)\left(t q^{2}-1\right) & \\
* & * & * \\
* & * & *
\end{array}\right) .
$$

We can also see that $\left(t q^{3}-1\right) /\left(t q^{4}\left(t^{2} q^{6}\right)^{k}(1+q)\left(t q^{2}-1\right)\right) \neq 0$ for $(\theta, \alpha) \in G$.

(3) If for $1 \leq i<2 n$, we have that

$$
r_{i}= \begin{cases}1, & i \text { is odd } \\ 2, & i \text { is even }\end{cases}
$$


then

$$
u=\left(S J^{1} S J^{2}\right)^{n-1} S J^{1} S J^{-3 n-[3(n-1)+1]}=\left(S J^{1} S J^{2}\right)^{n-1} S J^{1} S J^{-3(2 n-1)-1} .
$$

Since $J^{-3} \in Z\left(B_{3}\right)$, it follows that $u=\left(S J^{-2} S J^{-1}\right)^{n-1} S J^{-2} S J^{-1}$ and so $u=\left(S J^{-2} S J^{-1}\right)^{n}$. The eigenvalues of $K\left(S J^{-2} S J^{-1}\right)$ are $e_{1}=1, e_{2}=(a-\sqrt{b}) / 2 t q^{2}, e_{3}=(a+\sqrt{b}) / 2 t q^{2}$, where $a=1+t q^{2}+t^{2} q^{4}-q(1+t)-t q^{3}(1+t)$ and $b=-4 t^{2} q^{4}+\left(1+q(-1+(q-1) t)\left(1+t q^{2}\right)\right)^{2}$. These eigenvalues are distinct for $(\theta, \alpha) \in G$. Now, we diagonalize $K\left(S J^{-2} S J^{-1}\right)$ by a matrix $L$ given by

$$
L=\left(\begin{array}{ccc}
q & \frac{q(-c-\sqrt{b})}{-c+\sqrt{b}} & -\frac{q(-c+\sqrt{b})}{c+\sqrt{b}} \\
-1+q+\frac{1}{t q} & \frac{-2 q+2(q-1)^{2} t^{2} q^{3}}{-c+\sqrt{b}} & -\frac{-2 q+2(q-1)^{2} t^{2} q^{3}}{c+\sqrt{b}} \\
1 & 1 & 1
\end{array}\right)
$$

where $b$ is defined above and $c=1+t q^{2}+t q^{3}(t-1)-t^{2} q^{4}-q(1+t)$.

Also, we have that $\operatorname{det}(L)=\left(\left(-1+q+q\left(1+q+q^{2}\right) t-(q-1) t^{2} q^{3}\right) \sqrt{b}\right) /\left(q^{3}(1+(q-1) q) t^{3}\right) \neq 0$ when

$$
\theta \neq \pm \frac{\pi}{3}, \quad b \neq 0, \quad 1+2 \cos \theta+2 \cos (\theta+\alpha)-2 \cos (2 \theta+\alpha) \neq 0 .
$$

Then

$$
L^{-1} K\left(\left(S J^{-2} S J^{-1}\right)^{n}\right) L=\left(\begin{array}{ccc}
1 & 0 & 0 \\
0 & e_{2}^{n} & 0 \\
0 & 0 & e_{3}^{n}
\end{array}\right)
$$

If $e_{3}^{n}=1$, then $e^{i \beta n}=1$, where $\beta=\arg \left(e_{3}\right)$. This implies that $\beta / \pi \in \mathbb{Q}$, which contradicts the fact that $(\theta, \alpha) \in G$. Therefore, $L^{-1} K\left(\left(S J^{-2} S J^{-1}\right)^{n}\right) L \neq I$ and so $K\left(\left(S J^{-2} S J^{-1}\right)^{n}\right) \neq I$. We conclude that $u=\left(S J^{-2} S J^{-1}\right)^{n} \notin$ Ker $K$.

(4) If for $1 \leq i<2 n$, we have that

$$
r_{i}= \begin{cases}2, & i \text { is odd } \\ 1, & i \text { is even }\end{cases}
$$

then

$$
u=\left(S J^{2} S J^{1}\right)^{n-1} S J^{2} S J^{-3 n-[3(n-1)+2]}=\left(S J^{2} S J^{1}\right)^{n-1} S J^{2} S J(-3(2 n-1)-2) .
$$

Since $J^{-3} \in Z\left(B_{3}\right)$, it follows that $u=\left(S J^{-1} S J^{-2}\right)^{n-1} S J^{-1} S J^{-2}$ and so $u=\left(S J^{-1} S J^{-2}\right)^{n}$. Using the properties of the trace, we have that $\operatorname{tr}(K(u))=\operatorname{tr}\left(K\left(\left(S J^{-2} S J^{-1}\right)^{n}\right)\right)$. By our result in case (3) and Theorem 3.4, we have that $\operatorname{tr}(K(u)) \neq 3$ and so $u=\left(S J^{-1} S J^{-2}\right)^{n} \notin$ Ker $K$. 
(5) Let $u=S J^{r_{1}} S J^{r_{2}} \cdots S J^{r_{2 n-1}} S J^{-3 n-\sum_{i=1}^{2 n-1} r_{i}}$ such that not all $r_{i}$ 's are 1 and not all $r_{i}{ }^{\prime}$ s are 2. We also assume that there exists an integer $j$ such that $r_{j}=r_{j+1}$, where $1 \leq j<2 n$. We write $u=A J^{-p}$, where $A=S J^{r_{1}} S J^{r_{2}} \ldots S J^{r_{2 n-1}} S$ and $p=3 n+\sum_{i=1}^{2 n-1} r_{i}$. Without loss of generality, we assume that $K(A)$ and $K\left(J^{-p}\right)$ do not commute for some values of $q$ and $t$ in $G$. For this reason, we require that $p \equiv 1$ or $2(\bmod 3)$. If $K(A)$ and $K\left(J^{-p}\right)$ happened to commute for some values of $q$ and $t$ then we change the values of $q$ and $t$, slightly in a way that $(\theta, \alpha)$ is still in $G$ and the matrices $K(A), K\left(J^{-p}\right)$ do not commute anymore. This is due to the fact that $(q, t)$ belongs to a dense subset of $G$ by the hypothesis of the theorem.

The eigenvalues of $J^{-1}$ are $1 /\left(q^{2} t^{2 / 3}\right),-(-1)^{1 / 3} /\left(q^{2} t^{2 / 3}\right)$ and $(-1)^{2 / 3} /\left(q^{2} t^{2 / 3}\right)$. It is clear that these eigenvalues are distinct. We diagonalize $K\left(J^{-1}\right)$ by a matrix $W$ given by

$$
\left(\begin{array}{ccc}
q^{2} t^{2 / 3} & (-1)^{2 / 3} q^{2} t^{2 / 3} & (-1)^{4 / 3} q^{2} t^{2 / 3} \\
q\left(1+(q-1) t^{1 / 3}\right) t^{1 / 3} & (-t)^{1 / 3} q\left(-1+(-1)^{1 / 3}(q-1) t^{1 / 3}\right) & t^{1 / 3} q\left(1+(-1)^{2 / 3}(q-1)\right) t^{1 / 3} \\
1 & 1 & 1
\end{array}\right)
$$

Under direct computations, we see that $\operatorname{det}(W)=-3 i \sqrt{3} q^{3} t \neq 0$. Here $i=\sqrt{-1}$. Then we get that

$$
W^{-1} K\left(J^{-1}\right) W=\left(\begin{array}{ccc}
\frac{1}{q^{2} t^{2 / 3}} & 0 & 0 \\
0 & -\frac{(-1)^{1 / 3}}{q^{2} t^{2 / 3}} & 0 \\
0 & 0 & \frac{(-1)^{2 / 3}}{q^{2} t^{2 / 3}}
\end{array}\right) \text {. }
$$

We have that

$$
W^{-1} K(u) W=\left(W^{-1} K(A) W\right)\left(W^{-1} K\left(J^{-p}\right) W\right) .
$$

Assume, to get contradiction, that $u \in \operatorname{Ker}(K)$. Then

$$
W^{-1} K(A) W=\left(W^{-1} K(u) W\right)\left(W^{-1} K\left(J^{-p}\right) W\right)^{-1}=\left(W^{-1} K\left(J^{-p}\right) W\right)^{-1} .
$$

It follows that $W^{-1} K(A) W$ is a diagonal matrix. Therefore, $W^{-1} K(A) W$ and $W^{-1} K\left(J^{-p}\right) W$ commute, that is,

$$
W^{-1} K(u) W=W^{-1} K\left(J^{-p}\right) K(A) W .
$$

Then

$$
K(u)=K\left(J^{-p}\right) K(A) .
$$

Therefore, $K(A)$ and $K\left(J^{-p}\right)$ commute, which is a contradiction. 


\section{References}

[1] J. S. Birman, Braids, Links, and Mapping Class Groups, vol. 82, Princeton University Press, Princeton, NJ, USA, 1974.

[2] D. Krammer, "The braid group $B_{4}$ is linear," Inventiones Mathematicae, vol. 142, no. 3, pp. 451-486, 2000.

[3] S. J. Bigelow, "Braid groups are linear," Journal of the American Mathematical Society, vol. 14, no. 2, pp. 471-486, 2001.

[4] D. Krammer, "Braid groups are linear," Annals of Mathematics. Second Series, vol. 155, no. 1, pp. 131-156, 2002.

[5] R. D. Budney, "On the image of the Lawrence-Krammer representation," Journal of Knot Theory and its Ramifications, vol. 14, no. 6, pp. 773-789, 2005.

[6] M. N. Abdulrahim and M. Al-Tahan, "Complex specializations of Krammer's representation of the braid group, $B_{3}$," Journal of Mathematics and Statistics, vol. 4, no. 4, pp. 213-216, 2008.

[7] S. Albeverio and S. Rabanovich, "On a class of unitary representations of the braid groups $B_{3}$ and $B_{4}$," Institute for Applied Mathematics, vol. 611, 2007.

[8] E. Artin, "Theorie der zöpfe," Abhandlungen Aus Dem Mathematischen Seminar Der Universitat Hamburg, vol. 4, pp. 47-72, 1926. 


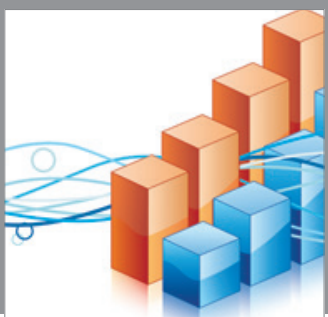

Advances in

Operations Research

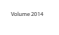

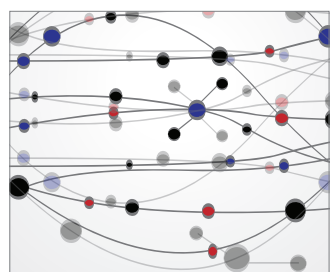

\section{The Scientific} World Journal
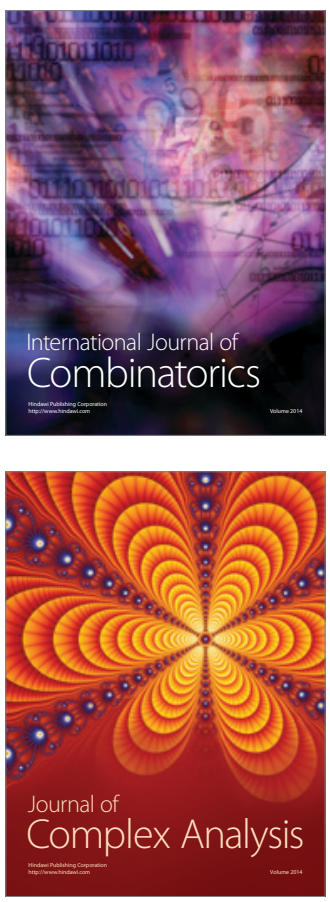

International Journal of

Mathematics and

Mathematical

Sciences
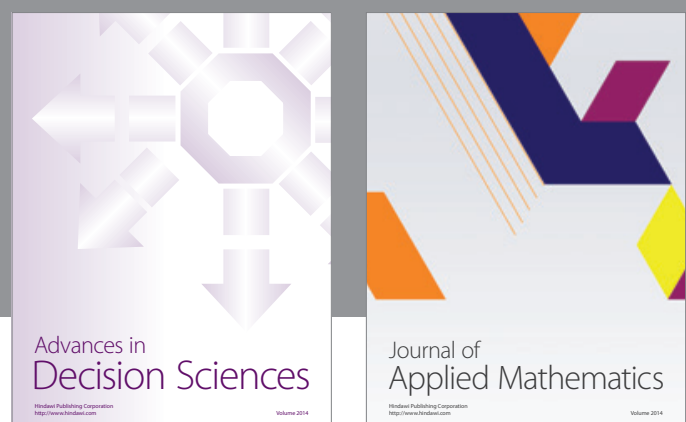

Journal of

Applied Mathematics
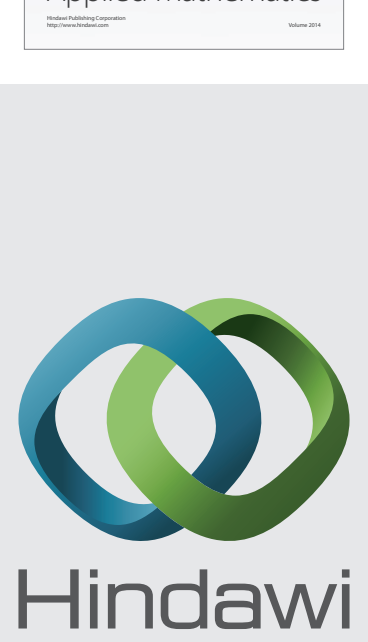

Submit your manuscripts at http://www.hindawi.com
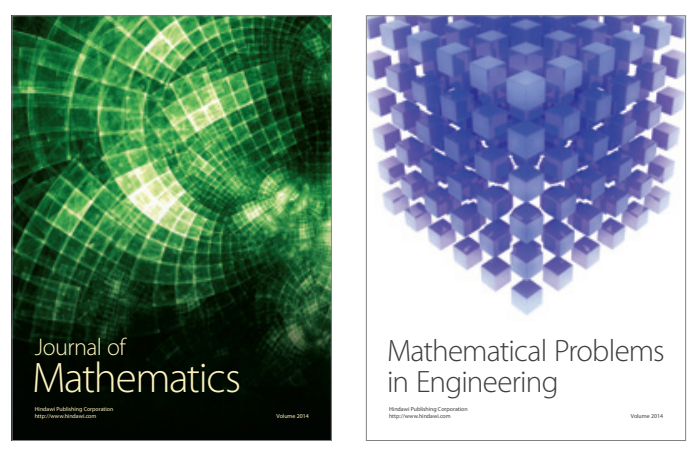

Mathematical Problems in Engineering
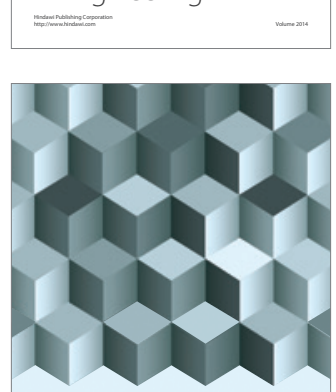

Journal of

Function Spaces
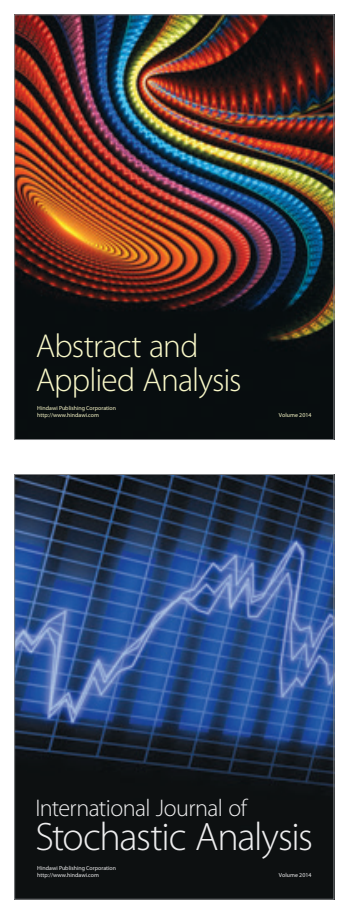

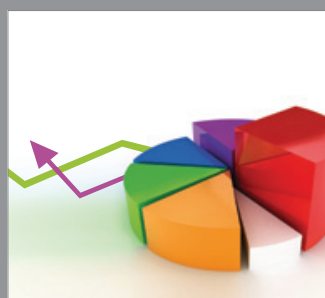

ournal of

Probability and Statistics

Promensencen
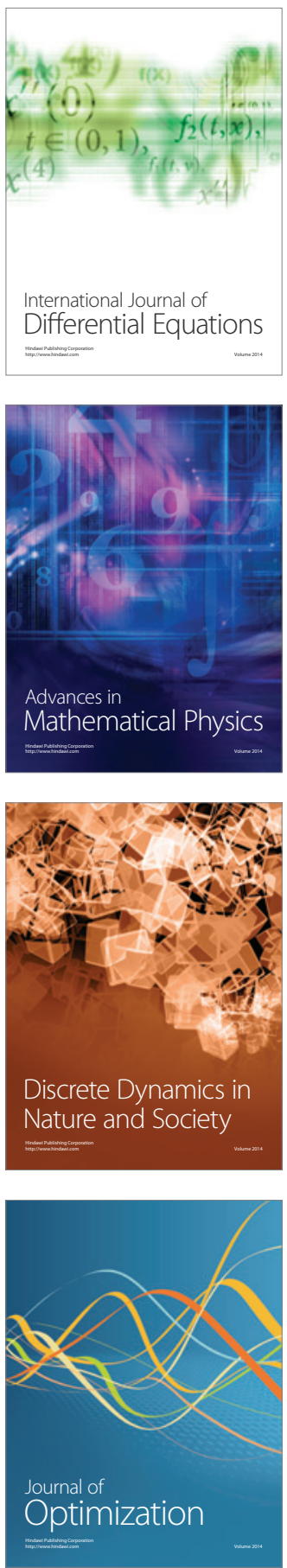